\title{
Arterial hypertension as a risk factor for myocardial infarction with non-obstructive coronary arteries (MINOCA)
}

\author{
Patryk Buller', Szymon Rosiak', Wojciech Rosiak ${ }^{3}$, Maciej Tyczyński ${ }^{4}$, Robert J. Gil ${ }^{4,5}$, Jacek Bil ${ }^{5}$ \\ 'Department of Cardiology, Voivodeship Hospital, Płock, Poland \\ ${ }^{2}$ Medical University of Gdańsk, Gdańsk, Poland \\ 3Internal Medicine Ward, District Hospital, Zuromin, Poland \\ ${ }^{4}$ Department of Invasive Cardiology, Central Clinical Hospital of the Ministry of Interior and Administration, Warsaw, Poland \\ ${ }^{5}$ Department of Invasive Cardiology, Centre of Postgraduate Medical Education, Warsaw, Poland
}

\begin{abstract}
Myocardial infarction with non-obstructive coronary arteries (MINOCA) as a relatively new disease entity distinguished from the group of acute coronary syndromes (ACS) is not a rare clinical problem and it requires in-depth diagnostics. MINOCA accounts for $5-10 \%$ of all ACS cases. MINOCA is most common between the ages of 50-60 and predominates in females. Coronary microvascular dysfunction and coronary vasospasm are among the potential mechanisms. The latest guidelines for the treatment of ACS in patients presenting without persistent ST-segment elevation emphasize the importance of searching for the causes of angina in patients with insignificant lesions in the coronary arteries by extending invasive diagnostics (e.g., acetylcholine provocation test) and using noninvasive diagnostics (e.g., CMR or SPECT). In the context of MINOCA, among the typical risk factors for coronary artery disease, arterial hypertension (HTN) seems to be the most important by inducing coronary microcirculation remodeling (mostly hypertrophy) and hence the narrowing of the lumen. Studies comparing patients with MINOCA and obstructive coronary artery disease (MI-CAD) in the context of risk factors, in particular HTN, were analyzed. In five out of nine analyzed studies, HTN occurred significantly more often in patients with MINOCA compared to patients with MI-CAD. The current pharmacotherapy recommendations focus on slowing the progression of coronary microvascular dysfunction (CMD), i.e., adequate treatment of risk factors and comorbidities, such as HTN. Therefore, it seems reasonable to conduct studies directly analyzing the relationship between HTN and MINOCA in order to improve diagnostics and establish appropriate pharmacotherapy that will improve prognosis.
\end{abstract}

Key words: MINOCA; acetylcholine; hypertensive heart disease; coronary artery remodeling

$$
\text { Arterial Hypertens. 2021, vol. 25, no. 3, pages: 106-111 }
$$

DOI: $10.5603 / A H . a 2021.0009$

\section{Introduction}

Myocardial infarction with non-obstructive coronary arteries (MINOCA) is a common and clinically important medical problem in the era of widespread use of coronary angiography. In the majority of studies on MINOCA, the analyzed populations of patients are dominated by females with an average age of 50-60 years. Compared to acute coronary syndrome (ACS) with obstructive coronary artery

Address for correspondence: Patryk Buller, MD, Department of Cardiology, Voivodeship Hospital in Plock, Plock, Poland, Medyczna Street 19, 09-400 Poland, tel: +48 2436467 61; e-mail: patryk@buller.com.pl

This article is available in open access under Creative Common Attribution-Non-Commercial-No Derivatives 4.0 International (CC BY-NC-ND 4.0) license, allowing to download articles and share them with others as long as they credit the authors and the publisher, but without permission to change them in any way or use them commercially 
disease $(\mathrm{CAD})$, patients are less burdened with traditional risk factors such as diabetes, dyslipidemia, and smoking [1]. It is estimated that MINOCA accounts for $5-10 \%$ of all ACS cases [2]. MINOCA is more common as non-ST-elevation myocardial infarction (NSTEMI) than ST-elevation myocardial infarction (STEMI). At the same time, in-hospital mortality is lower compared to patients with obstructive coronary artery disease (MI-CAD) [3].

The pathomechanism of MINOCA is not fully understood, and the underlying cause of the disease appears to be multifactorial. One of the potentially important risk factors is arterial hypertension (HTN). In this review, we aimed to summarize the impact of HTN on MINOCA pathomechanisms as well as outcomes.

\section{Etiopathogenesis}

The pathogenesis of MINOCA appears to be varied. Eccentric plaques with positive remodeling can be prone to significant instability. Plaque disruption is followed by local thrombosis and then fibrinolysis, which may result in distal embolization. An angiographic image may suggest plaque rupture, but a final diagnosis can be made using higher-resolution optical coherence tomography (OCT) or, to a lesser extent, intravascular ultrasound (IVUS) [4]. Hereditary (e.g., factor V Leiden, von Willebrand factor) and acquired (thrombotic thrombocytopenia purple TTP, the autoimmune disorder antiphospholipid syndrome, heparin-induced thrombocytopenia HIT) states may also predispose to coronary thrombosis or embolism. The small size myocardial infarction is best visualized in cardiac magnetic resonance (CMR).

Spontaneous dissection of the coronary artery (SCAD) is a rare mechanism of MINOCA but is not rarely observed as a cause of ACS in women under 50 years of age. SCAD causes a significant obstruction of flow in most cases, but occasionally the vessels may appear normal due to the gradual narrowing of the distal artery lumen, indicating a possible cause of MINOCA [5].

The main pathophysiological mechanisms responsible for microvascular angina symptoms are coronary microvascular dysfunction (CMD) and vasospastic angina (VSA). They are considered primarily responsible for ischemia with non-obstructive coronary arteries (INOCA) [6] and may manifest as MINOCA.

In the case of CMD, there is a remodeling of small arteries and arterioles. Hyperplasia and hy- pertrophy change the smooth muscle system causing narrowing of the vessel lumen, which results in the reduction of the coronary reserve with clinically manifested angina [7]. Also, microcirculatory spasm might be responsible for CMD. It has been suggested that changes in microcirculation may precede those manifested in large vessels [8]. Arterial hypertension (HTN) is a crucial risk factor inducing the abovementioned pathological processes [9].

Coronary vasospasm (CVsp) is a broad subject involving structural $\mathrm{CAD}$ but may also coexist with CMD. Gender is a potent modifier of CVsp as the cause of INOCA is more common in men than in women [10]. Interestingly, it has also been noted that HTN is paradoxically negatively related to CVsp. This finding suggests that the pathogenesis of CVsp is distinct from that of coronary atherosclerosis [11].

Spontaneous CVsp is rare during routine coronary angiography. And provocation tests are usually performed in tertiary centers, mainly with the use of intracoronary acetylcholine or ergonovine (intravenous administration is also possible but not recommended due to the high risk of death). Acetylcholine is injected intracoronary in gradually increasing doses. Graphs made on an ongoing basis show the extent of the contraction and its nature (diffuse or focal). Lumen reductions of $90 \%$ comparing to the reference after administration of nitroglycerin are interpreted as positive. The detailed diagnostic criteria were prepared by COVADIS (Coronary Vasomotor Disorders International) [12]. The joined use of adenosine and acetylcholine is used to assess endothelial independent and dependent dysfunction to differentiate between CMD and CVsp. After intracoronary administration of adenosine, the artery relaxes to its maximum. Coronary flow reserve $(\mathrm{CFR}) \leq 2.0$ in response to adenosine can diagnose endothelial-independent and endothelial-dependent CMD. Another invasive indicator is the index of microvascular resistance (IMR), which uses the phenomenon of thermodilution with the help of a wire with a pressure and temperature sensor. Values $\geq 25$ $\mathrm{U}$ in the absence of epicardial stenosis indicate a disturbance in blood flow through the microcirculation vessels [13]. Importantly, this parameter is independent of hemodynamics, preload, afterload, and heart rate [14].

It is worth adding that the performance of these tests (i.e., CFR, IMR, provocative test with acetylcholine) during one procedure does not significantly extend the duration of the procedure but provides a complete set of relevant data [15]. In addition, when performing provocation tests, the extension of imaging diagnostics through the use of IVUS or 
OCT may, apart from the diagnosis of CVsp, visualize thrombus, vulnerable plaque, or SCAD, which forces the implementation of appropriate therapeutic measures $[4,16]$.

The AChPOL register examined, among other things, the feasibility and safety of provocative test with acetylcholine (AChT). Of the group of qualified patients $(n=211), 99$ had revealed variant angina, and 72 patients had a coronary microvascular spasm. During the 5-year follow-up, the group of patients with MINOCA and confirmed microvascular contraction had more frequent recurrences of angina and required hospitalization more often compared to patients with negative AChT. The study confirmed the popularization of AChT, which will increase the detection of CVsp, especially in patients with MINOCA, and thus improve their prognosis [17].

Gender is a strong modifier of CVsp as the cause of INOCA is more common in men than in women [10]. Interestingly, it has also been noted that HTN is paradoxically negatively related to CVsp. This finding suggests that the pathogenesis of CVsp is distinct from that of coronary atherosclerosis [11].

\section{MINOCA in light of the latest guidelines}

A new subsection on MINOCA has been included in the latest ESC Guidelines for the management of acute coronary syndromes in patients presenting without persistent ST-segment elevation. Fundamental to the diagnosis of MINOCA is cardiac troponin $>99^{\text {th }}$ percentile of the upper reference level, but such rise of troponin may also be related to non-ischemic mechanisms. Based on the definition of ESC Fourth Universal Definition of Myocardial Infarction, myocarditis, cardiomyopathy, and Takotsubo syndrome do not meet the criteria for the diagnosis of MINOCA [18]. Local wall contractility abnormalities may indicate an epicardial MINOCA cause or other specific causes that require further diagnosis. The essential tool in the differential diagnosis is CMR, which makes it possible to identify the cause in as many as $87 \%$ of MINOCA patients. Late enhancement after gadolinium administration in the sub-endocardium suggests an ischemic cause, while the sub-epicardial localization may indicate cardiomyopathies or myocarditis [16].

In July 2020, the position of the expert group of the European Association of Percutaneous Cardiovascular Interventions (EAPCI) on INOCA was published. The document emphasizes that angina is the most common CAD symptom. The lack of confirmation in routine diagnostics should lead cli- nicians to differentiate INOCA end-types before non-cardiac causes of chest discomfort.

In the search for the cause of impaired myocardial perfusion and ischemia, noninvasive tests, such as transthoracic Doppler echocardiography (TTDE), positron emission tomography (PET), single photon emission computed tomography (SPECT), or the CMR, as mentioned above, may be helpful. If INOCA is suspected during invasive diagnostics, additional diagnostic procedures (for example, pressure and flow measurements and pharmacological coronary reactivity testing) should be implemented to distinguish the mechanism of CMD from vasospastic angina or non-cardiac pain. Moreover, it is recommended to perform fractional flow reserve (FFR) in patients with borderline stenosis in whom angioplasty is considered to be a procedure with a high benefit rate for the patient [6].

\section{Correlation between hypertension and coronary artery disease}

From 1990 to 2019, the number of adults worldwide suffering from hypertension increased from 2.18 billion to 4.06 billion, and the number of deaths from hypertension increased from 6.79 million to 10.8 million [19]. Arterial hypertension is associated with an ongoing age-related risk of death from ischemic heart disease [20]. The correlation between HTN and MINOCA as a relatively new disease entity is important in establishing the most optimal pharmacotherapy for the patient, reducing angina symptoms, and improving prognosis.

The frequently coexisting MINOCA risk factors such as aging, diabetes, and dyslipidemia lead to increased arterial stiffness and increased pulse pressure. HTN affects the remodeling of small arteries, causing their hypertrophy, narrowing the lumen, and reducing blood vessels density. This contributes to CMD [9]. The effect of lowering blood pressure on improving CMD was studied by Mizuno et al. in 2012. Patients with similar CFR results were enrolled in the study and reassessed after a 12-month treatment period with antihypertensive drugs. The analysis showed that lowering blood pressure is an independent determinant of improvement in CFR. It is noteworthy that excessive reduction of diastolic blood pressure weakened this effect [21].

In the aforementioned AChPOL Registry, a group of 45 patients with MINOCA was identified (including 19 in the variant angina group, 23 in the microvascular spasm group, and three in the negative AChT group). Compared to patients without 
Table 1. Comparison of the prevalence of arterial hypertension in patients with MINOCA and MI-CAD in individual studies

\begin{tabular}{|l|c|c|c|}
\hline Trial & MINOCA & MI-CAD & p value \\
\hline & $\%$ & $\%$ & 0.001 \\
\hline Eggers et al. [22] & 41.1 & 43.2 & $>0.05$ \\
\hline Bil et al. [13] & 52 & 45 & 0.037 \\
\hline Grodzinsky et al.[23] & 68.8 & 63.4 & $<0.05$ \\
\hline Jánosi et al. [24] & 73.7 & 79.1 & 0.029 \\
\hline Abdu et al. [25] & 52.3 & 62.4 & $<0.0001$ \\
\hline Rakowski et al. [26] & 56.4 & 64.3 & $>0.05$ \\
\hline Lopez-Pais et al. [27] & 61.5 & 62.1 & $>0.05$ \\
\hline Raparelli et al. [28] & 32.9 & 36.9 & $>0.05$ \\
\hline Vranken et al. [29] & 48.6 & 43.2 & \\
\hline
\end{tabular}

a history of MINOCA, HTN was significantly more common in the MINOCA group ( $82.2 \%$ vs. $57.2 \%$, $\mathrm{p}=0.02$ ). The study (as very promising) is definitely worth doing on a more extensive research sample, which would lead to an increased ability to detect differences [17].

The literature search yielded nine recent studies (including one meta-analysis covering 27 large clinical trials/registries) attempting to characterize MINOCA in the context of the clinical profile, prognosis, and MACE rates. The results were compared with those of patients with MI-CAD. The general studies' conclusions are common and indicate that the prognosis of patients is worse than previously believed, mainly in the context of cardiovascular events. In addition, each study identified HTN as a risk factor. The incidence of HTN in the MINOCA group in the analyzed studies ranged from $41.1 \%$ to $73.7 \%$. It is worth adding that in 5 out of 9 analyzed studies, $\mathrm{HTN}$ as a risk factor occurred more frequently in MINOCA than in MI-CAD. The results are summarized in Table 1.

Worth mentioning is also the study by Carrick et al., in which the pathophysiology of damage to the coronary microcirculation in patients with STEMI due to HTN was analyzed. Invasive and noninvasive reperfusion injury tests, circulating markers of inflammation, serial imaging of infarct pathology, and remodeling were conducted. HTN was not associated with acute reperfusion injury, as shown by direct angiographic measurements (TIMI frame count, TIMI blush grade). A potential explanation comes from taking antihypertensive therapies with vasoprotective effect angiotensin-converting enzyme inhibitors (ACE-I). However, the study results provided evidence that patients with HTN (as an independent risk factor) and acute STEMI had a worse prognosis due to microvascular injury within the infarct zone.
Clinically, this was manifested by more frequent hospitalizations for heart failure [30].

\section{Treatment}

The management of INOCA can be broken down into the influence of risk factors and antianginal medication. In patients with MINOCA, as in most patients with $\mathrm{CAD}$, it is important to modify risk factors related to lifestyle in order to improve its quality and prognosis. Behavioral interventions can be carried out by nutritionists (low-fat, low-calorie diets), psychologists (coping with stress, obesity), nurses, or doctors [6].

The next stage of treatment is pharmacotherapy. Of the main risk factors for CAD, i.e., HTN, dyslipidemia, smoking, and diabetes, the main goal of treatment is adequate blood pressure control. This is due to a reduction in CMD progression and a reduction in the occurrence of angina symptoms. It is not easy as the MINOCA group of patients is often heterogeneous and diverse. That group of patients experience angina symptoms at least as frequently as patients after MI-CAD, and as they are not by definition candidates for revascularization, appropriate pharmacotherapy is paramount [23].

According to the latest reports, the use of beta-blockers is associated with a significantly lower risk of mortality and major cardiovascular events. In contrast, the use of aspirin has a potentially detrimental effect on prognosis. These data seem to be important because standard therapy in secondary prevention in patients after ACS with non-obstructive CAD may be unfavorable or even harmful for some patients. This is another argument forcing large-scale research to be carried out in the future to precisely characterize this disease entity [36]. 
In relation to the $2018 \mathrm{ESC} / \mathrm{EHA}$ guidelines for the treatment of HTN, the main medications in patients with symptomatic angina are beta-blockers, for example, nebivolol $2.5-10 \mathrm{mg}$ daily dose (reduction of myocardial oxygen consumption, antioxidant properties) and calcium channel blockers, for instance, amlodipine $10 \mathrm{mg}$ daily (vascular smooth muscle relaxation, reduction in myocardial oxygen consumption) [31]. The study by Neglia et al. from 2011 confirmed the important role of using ACE-I in therapy, i.e., improvement in hyperemic myocardial blood flow in hypertensive microvascular angina patients [32].

According to the EAPCI expert consensus, the use of ACE-I/ARB improves CFR in CMD. It should be noted that these drugs are easily combined in a single formulation with both beta-blockers and calcium channel blockers. The dominant role in the treatment of microvascular angina caused by CMD is played by to be beta-blockers and calcium channel blockers (in the next stage - nicorandil, ranolazine, ivabradine, trimetazidine). In patients with $\mathrm{CV}$ sp (after acetylcholine testing), calcium antagonists (e.g. high-dose diltiazem) should be considered as first-line treatment. Short-acting nitrates may require frequent repeated doses. On the other hand, the use of short-acting nitrates is often poorly tolerated, and it is believed that may increase symptoms in patients with microvascular angina. In patients with non-obstructive $\mathrm{CAD}$, statins may also be effective. Moreover, in patients with CVsp and reduced CFR they exhibit anti-inflammatory properties [6].

In the summary of pharmacotherapy, it is also worth mentioning the ongoing study MINOCA-BAT. The primary objective of the study is to determine whether beta-blockade compared with no beta blockade and ACE-I / ARB compared with no ACE-I / ARB have an influence on lowering the occurrence of composite endpoint (i.e. death, rehospitalization for AMI, ischemic stroke, and heart failure). Though it has been long known that patients with MINOCA are at greater risk of cardiovascular events, so far no benefits from the use of secondary prophylactic therapies have been evaluated in randomized trials. The results of MINOCA-BAT study may have an impact on guidelines for management of MINOCA [33].

\section{Conclusions}

MINOCA is still a new and often underestimated issue in cardiology. More research is needed to clarify the disease's pathophysiology, improve diagnostics, and determine the appropriate, effective, and adequate pharmacotherapy. It seems justified to conduct studies directly analyzing the relationship between arterial hypertension and MINOCA as an important etiological factor influencing this disease's pathomechanism.

\section{References}

1. Pizzi C, Xhyheri B, Costa GM, et al. Nonobstructive Versus Obstructive Coronary Artery Disease in Acute Coronary Syndrome: A Meta-Analysis. J Am Heart Assoc. 2016; 5(12), doi: 10.1161/ JAHA.116.004185, indexed in Pubmed: 27986756.

2. Lindahl B, Baron T, Erlinge D, et al. Medical Therapy for Secondary Prevention and Long-Term Outcome in Patients With Myocardial Infarction With Nonobstructive Coronary Artery Disease. Circulation. 2017; 135(16): 1481-1489, doi: 10.1161/CIRCULATIONAHA.116.026336, indexed in Pubmed: 28179398.

3. Smilowitz NR, Mahajan AM, Roe MT, et al. Mortality of Myocardial Infarction by Sex, Age, and Obstructive Coronary Artery Disease Status in the ACTION Registry-GWTG (Acute Coronary Treatment and Intervention Outcomes Network Registry-Get With the Guidelines). Circ Cardiovasc Qual Outcomes. 2017; 10(12): e003443, doi: 10.1161/CIRCOUTCOMES.116.003443, indexed in Pubmed: 29246884.

4. Bil J, Pawlowski T, Gil RJ. Coronary spasm revascularized with a bioresorbable vascular scaffold. Coron Artery Dis. 2015; 26(7): 634-636, doi: 10.1097/MCA.0000000000000297, indexed in Pubmed: 26267749.

5. Tamis-Holland JE, Jneid H, Reynolds HR, et al. American Heart Association Interventional Cardiovascular Care Committee of the Council on Clinical Cardiology; Council on Cardiovascular and Stroke Nursing; Council on Epidemiology and Prevention; and Council on Quality of Care and Outcomes Research. Contemporary Diagnosis and Management of Patients With Myocardial Infarction in the Absence of Obstructive Coronary Artery Disease: A Scientific Statement From the American Heart Association. Circulation. 2019; 139(18): e891-e908, doi: 10.1161/ CIR.0000000000000670, indexed in Pubmed: 30913893.

6. Kunadian V, Chieffo A, Camici PG, et al. An EAPCI Expert Consensus Document on Ischaemia with Non-Obstructive Coronary Arteries in Collaboration with European Society of Cardiology Working Group on Coronary Pathophysiology \& Microcirculation Endorsed by Coronary Vasomotor Disorders International Study Group. EuroIntervention. 2021; 16(13): 1049-1069, doi: 10.4244/EIJY20M07_01, indexed in Pubmed: 32624456.

7. Kern A, Górny J, Zaleska M, et al. Coronary microcirculation dysfunction in patients with arterial hypertension. Arterial Hypertension. 2018; 22(4): 151-155, doi: 10.5603/ah.a2018.0003.

8. Labazi H, Trask AJ. Coronary microvascular disease as an early culprit in the pathophysiology of diabetes and metabolic syndrome. Pharmacol Res. 2017; 123: 114-121, doi: 10.1016/j. phrs.2017.07.004, indexed in Pubmed: 28700893.

9. Bairey Merz CN, Pepine CJ, Walsh MN, et al. Ischemia and No Obstructive Coronary Artery Disease (INOCA): Developing Evidence-Based Therapies and Research Agenda for the Next Decade. Circulation. 2017; 135(11): 1075-1092, doi: 10.1161/CIRCULATIONAHA.116.024534, indexed in Pubmed: 28289007.

10. Hung MY, Hsu KH, Hung MJ, et al. Interactions among gender, age, hypertension and $\mathrm{C}$-reactive protein in coronary vasospasm. Eur J Clin Invest. 2010; 40(12): 1094-1103, doi: 10.1111/j.13652362.2010.02360.x, indexed in Pubmed: 20718850.

11. Sugiishi M, Takatsu F. Cigarette smoking is a major risk factor for coronary spasm. Circulation. 1993; 87(1): 76-79, doi: 10.1161/01.cir.87.1.76, indexed in Pubmed: 8419026.

12. Beltrame JF, Crea F, Kaski JC, et al. Coronary Vasomotion Disorders International Study Group (COVADIS). International standardization of diagnostic criteria for vasospastic angina. Eur 
Heart J. 2017; 38(33): 2565-2568, doi: 10.1093/eurheartj/ ehv351, indexed in Pubmed: 26245334.

13. Bil J, Pietraszek N, Pawlowski T, et al. Advances in Mechanisms and Treatment Options of MINOCA Caused by Vasospasm or Microcirculation Dysfunction. Curr Pharm Des. 2018; 24(4): 517-531, doi: 10.2174/1381612824666180108121253, indexed in Pubmed: 29308736.

14. Pietraszek N, Możeńska O, Bil J. Invasive assessment of coronary microcirculation in patients with ST-segment elevation myocardial infarction. Kardiol Inwazyjna. 2017; 12(4): 18-24.

15. Bil J, Tyczyński M, Modzelewski P, et al. Acetylcholine provocation test with resting full-cycle ratio, coronary flow reserve, and index of microcirculatory resistance give definite answers and improve health-related quality of life. Kardiol Pol. 2020; 78(12): 1291-1292, doi: 10.33963/KP.15619, indexed in Pubmed: 32975096.

16. Collet JP, Thiele H, Barbato E, et al. ESC Scientific Document Group . 2020 ESC Guidelines for the management of acute coronary syndromes in patients presenting without persistent ST-segment elevation. Eur Heart J. 2021; 42(14): 1289-1367, doi: 10.1093/eurheartj/ehaa575, indexed in Pubmed: 32860058.

17. Bil J, MoŻeŃska O, Segiet-ŚwiĘcicka A, et al. Revisiting the use of the provocative acetylcholine test in patients with chest pain and nonobstructive coronary arteries: A five-year follow-up of the AChPOL registry, with special focus on patients with MINOCA. Transl Res. 2021; 231: 64-75, doi: 10.1016/j.trsl.2020.11.009, indexed in Pubmed: 33232803.

18. Thygesen K, Alpert JS, Jaffe AS, et al. Executive Group on behalf of the Joint European Society of Cardiology (ESC)/American College of Cardiology (ACC)/American Heart Association (AHA)/ World Heart Federation (WHF) Task Force for the Universal Definition of Myocardial Infarction. Fourth Universal Definition of Myocardial Infarction (2018). Circulation. 2018; 138(20): e618-e651, doi: 10.1161/CIR.0000000000000617, indexed in Pubmed: 30571511.

19. Roth GA, Mensah GA, Johnson CO, et al. GBD-NHLBI-JACC Global Burden of Cardiovascular Diseases Writing Group. Global Burden of Cardiovascular Diseases and Risk Factors, 1990-2019: Update From the GBD 2019 Study. J Am Coll Cardiol. 2020; 76(25): 2982-3021, doi: 10.1016/j.jacc.2020.11.010, indexed in Pubmed: 33309175.

20. Lewington S, Clarke R, Qizilbash N, et al. Prospective Studies Collaboration. Age-specific relevance of usual blood pressure to vascular mortality: a meta-analysis of individual data for one million adults in 61 prospective studies. Lancet. 2002; 360(9349): 1903-1913, doi: 10.1016/s0140-6736(02)11911-8, indexed in Pubmed: 12493255.

21. Mizuno R, Fujimoto S, Saito Y, et al. Optimal antihypertensive level for improvement of coronary microvascular dysfunction: the lower, the better? Hypertension. 2012; 60(2): 326-332, doi: 10.1161/HYPERTENSIONAHA.111.189209, indexed in Pubmed: 22753208.

22. Eggers KM, Hjort M, Baron T, et al. Morbidity and cause-specific mortality in first-time myocardial infarction with nonobstructive coronary arteries. J Intern Med. 2019; 285(4): 419-428, doi: 10.1111/joim.12857, indexed in Pubmed: 30474313.
23. Grodzinsky A, Arnold SV, Gosch K, et al. Angina Frequency After Acute Myocardial Infarction In Patients Without Obstructive Coronary Artery Disease. Eur Heart J Qual Care Clin Outcomes. 2015; 1(2): 92-99, doi: 10.1093/ehjqcco/qcv014, indexed in Pubmed: 28239487.

24. Jánosi A, Ferenci T, Kőszegi Z, et al. [Myocardial infarction without obstructive coronary artery disease (MINOCA) — prevalence and prognosis]. Orv Hetil. 2019; 160(45): 1791-1797, doi: 10.1556/650.2019.31555, indexed in Pubmed: 31680539.

25. Abdu FA, Liu Lu, Mohammed AQ, et al. Myocardial infarction with non-obstructive coronary arteries (MINOCA) in Chinese patients: Clinical features, treatment and 1 year follow-up. Int J Cardiol. 2019; 287: 27-31, doi: 10.1016/j.ijcard.2019.02.036, indexed in Pubmed: 30826195.

26. Rakowski T, De Luca G, Siudak Z, et al. Characteristics of patients presenting with myocardial infarction with nonobstructive coronary arteries (MINOCA) in Poland: data from the ORPKI national registry. J Thromb Thrombolysis. 2019; 47(3): 462-466, doi: 10.1007/s11239-018-1794-z, indexed in Pubmed: 30565147.

27. Lopez-Pais J, Izquierdo Coronel B, Galán Gil D, et al. Clinical characteristics and prognosis of myocardial infarction with nonobstructive coronary arteries (MINOCA): A prospective singlecenter study. Cardiol J. 2020 [Epub ahead of print], doi: 10.5603/ CJ.a2020.0146, indexed in Pubmed: 33140385.

28. Raparelli V, Elharram M, Shimony A, et al. Myocardial Infarction With No Obstructive Coronary Artery Disease: Angiographic and Clinical Insights in Patients With Premature Presentation. Can J Cardiol. 2018; 34(4): 468-476, doi: 10.1016/j.cjca.2018.01.004, indexed in Pubmed: 29571427.

29. Vranken NPA, Pustjens TFS, Kolkman E, et al. MINOCA: The caveat of absence of coronary obstruction in myocardial infarction. Int J Cardiol Heart Vasc. 2020; 29: 100572, doi: 10.1016/j. ijcha.2020.100572, indexed in Pubmed: 32642553.

30. Carrick D, Haig C, Maznyczka AM, et al. Hypertension, Microvascular Pathology, and Prognosis After an Acute Myocardial Infarction. Hypertension. 2018; 72(3): 720-730, doi: 10.1161/HYPERTENSIONAHA.117.10786, indexed in Pubmed: 30012869.

31. Williams B, Mancia G, Spiering W, et al. ESC Scientific Document Group . 2018 ESC/ESH Guidelines for the management of arterial hypertension. Eur Heart J. 2018; 39(33): 3021-3104, doi: 10.1093/eurheartj/ehy339, indexed in Pubmed: 30165516.

32. Neglia D, Fommei E, Varela-Carver A, et al. Perindopril and indapamide reverse coronary microvascular remodelling and improve flow in arterial hypertension. J Hypertens. 2011; 29(2): 364-372, doi: 10.1097/HJH.0b013e328340a08e, indexed in Pubmed: 21045728.

33. Nordenskjöld AM, Agewall S, Atar D, et al. Randomized evaluation of beta blocker and ACE-inhibitor/angiotensin receptor blocker treatment in patients with myocardial infarction with non-obstructive coronary arteries (MINOCA-BAT): Rationale and design. Am Heart J. 2021; 231: 96-104, doi: 10.1016/j. ahj.2020.10.059, indexed in Pubmed: 33203618. 